\title{
Effects of Nonlocal One-Pion-Exchange Potential in Deuteron
}

\author{
J. L. Forest田 \\ Jefferson Lab Theory Group, 12000 Jefferson Ave., Newport News, VA 23606
}

(November 13, 2018)

\begin{abstract}
The off-shell aspects of the one-pion-exchange potential (OPEP) are discussed. Relativistic Hamiltonians containing relativistic kinetic energy, relativistic OPEP with various off-shell behaviors and Argonne $v_{18}$ short-range parameterization are used to study the deuteron properties. The OPEP off-shell behaviors depend on whether a pseudovector or pseudoscalar pion-nucleon coupling is used and are characterized by a parameter $\mu$. We study potentials having $\mu=-1,0$ and +1 and we find that they are nearly unitarily equivalent. We also find that a nonrelativistic Hamiltonian containing local potentials and nonrelativistic kinetic energy provides a good approximation to a Hamiltonian containing a relativistic OPEP based on pseudovector pion-nucleon coupling and relativistic kinetic energy.
\end{abstract}

PACS numbers: 21.30.-x, 24.10.Jv

Typeset using REVTEX 


\section{INTRODUCTION}

The modern high-quality two-nucleon potential models include three local potentials: Nijmegen II [1], Reid93 [1] and Argonne $v_{18}$ (AV18) [2], and two nonlocal potential: Nijmegen I [1] and CD-Bonn [3]. These potentials accurately fit the $N N$ scattering data of the Nijmegen database [4] and the deuteron binding energy, and are essentially phase-equivalent. The three local potentials predict very similar deuteron $D$-state probability $P_{D}(5.70,5.64$ and $5.76 \%)$ and triton energy $(-7.63,-7.62$ and $-7.61 \mathrm{MeV})$ respectively, however the nonlocal CD-Bonn potential gives rather different results: $4.83 \%$ and $-8.00 \mathrm{MeV}$. The Nijmegen I predicts similar $P_{D}$ as the local Nijmegen II but slightly larger triton energy of $-7.72 \mathrm{MeV}$. The experimental value of triton energy is $-8.48 \mathrm{MeV}$, and $P_{D}$ is not an observable. One may naturally ask: why is CD-Bonn so unique among the modern potentials? Is one model better than the other, or are all modern potentials equally good in predicting properties of nuclei after correctly including all other ingredients such as three-body forces, relativistic effects, meson-exchange currents, etc.? What role do nonlocalities play in properties of light nuclei and nuclear matter?

To answer these questions, one has to look into the microscopic description of nuclear interactions. Theoretically as well as experimentally it is known that the one-pionexchange potential (OPEP) provides the long range part of the two-body potential $v_{N N}$. The intermediate- and short-range parts of $v_{N N}$ are obtained by fitting the experimental data. The three local potentials have the same nonrelativistic on-shell OPEP at long range, but different phenomenological parameterizations at intermediate and short range. Nijmegen I has nonlocalities in the central part of the potential at short range. CD-Bonn, however,

contains off-shell nonlocalities in OPEP predicted by pseudoscalar pion-nucleon coupling as well as nonlocalities in the phenomenological shorter range parts. The fact that the local potentials and the Nijmegen I model predict very similar deuteron properties which are very different from those predicted by CD-Bonn seems to suggest that differences in OPEP could be the main cause. 
Inspired by the above comparison among the modern potentials, we want to understand various aspects of OPEP off-shell nonlocalities in this work. The nonlocality in the shorterrange part of the potential may also play some role, but it is not our primary interest here.

There has always been some ambiguity about the off-shell effects in the two-body potentials. Friar [5] recently categorized these ambiguities into three types: (i) those caused by an energy-dependent potential which occur naturally when expanding energy denominators in Schrödinger perturbation theory; (ii) those arising from unitary transformations of field variables used in the Lagrangians; (iii) those due to different choices of relativistic Hamiltonians. In this work we are only interested in understanding the second type of ambiguities. In Friar's notation, potentials having different off-shell forms are characterized by parameters $\mu$ and $\nu$, where the $\mu$-dependence comes from whether using pseudoscalar (PS) or pseudovector (PV) relativistic pion-nucleon interactions, while the $\nu$-dependence comes from the retardation effects. Here we are only interested in the $\mu$-dependent off-shell behaviors, but neglect the retardation effects which seem to be relatively unimportant. In Friar's notation, we choose $\nu=1 / 2$ (no retardation) throughout this work.

The relativistic OPEP are identical on-shell, regardless of the assumed coupling (PS or PV), but they differ off-shell. There is no unique description for the off-shell behavior of relativistic OPEP. It depends upon a parameter $\mu$ whose common choices [5] are $\mu=-1$ (PS coupling) used in CD-Bonn [3], $\mu=0$ (minimal nonlocality) used in our earlier work [6], and $\mu=+1$ (PV coupling) favored by conventional Chiral Perturbation Theory (CPT). As suggested by Friar [7,8] two decades ago, two-body potentials differing in the value of $\mu$ are related by unitary transformations up to order $1 / \mathrm{m}^{2}$. All these potentials are correct to this order, and predict identical observables, even though they have different forms. In this view, when various two-body potentials are combined with their corresponding threebody potentials, the triton energy should be accurately predicted; when electromagnetic current operators are treated consistently with the potential, the electromagnetic observables should be independent of the choice of $\mu$ [9]. This is rather interesting and useful because if there indeed exists such unitary equivalence, then we could choose the simpler 
and computationally-easier local potentials together with their three-body forces, and consistent electromagnetic charge and current operators to study properties of light nuclei.

The purpose of this paper is to study deuteron properties with potentials containing $\mu=-1,0,+1$ relativistic OPEP (Sec. II), and to examine the unitary equivalence of these potentials (Sec III). We also attempt to find local potentials which would give a smaller value for deuteron $P_{D}$, characteristic of the $\mu=-1$ nonlocal potentials, but with no success (Sec IV). The conclusions are given in Sec. V. Some of the detailed derivations involved in this work are given in an Appendix.

\section{OFF-SHELL EFFECTS IN DEUTERON}

Consider a relativistic Hamiltonian

$$
H_{R}=2 \sqrt{p^{2}+m^{2}}-2 m+v_{\pi}+v_{R}
$$

in the frame in which the deuteron is at rest. The transformation of this $H_{R}$ to other frames is discussed in Ref. [10]. Here $v_{\pi}$ is the OPEP containing off-shell nonlocalities, and $v_{R}$ is the remaining part of the potential which is phenomenological. In this work we use AV18 parameterization for $v_{R}$. As suggested by Friar [5], after neglecting retardation effects, the relativistic OPEP can be expressed in the following general form

$$
\begin{aligned}
v_{\pi}^{\mu}\left(\mathbf{p}^{\prime}, \mathbf{p}\right)= & -\frac{f_{\pi N N}^{2}}{m_{\pi}^{2}} \frac{\boldsymbol{\tau}_{i} \cdot \boldsymbol{\tau}_{j}}{m_{\pi}^{2}+q^{2}} \frac{m}{E} \frac{m}{E^{\prime}}\left[\boldsymbol{\sigma}_{i} \cdot \mathbf{q} \boldsymbol{\sigma}_{j} \cdot \mathbf{q}\right. \\
& \left.+\mu \times\left(E^{\prime}-E\right)\left(\frac{\boldsymbol{\sigma}_{i} \cdot \mathbf{p}^{\prime} \boldsymbol{\sigma}_{j} \cdot \mathbf{p}^{\prime}}{E^{\prime}+m}-\frac{\boldsymbol{\sigma}_{i} \cdot \mathbf{p} \boldsymbol{\sigma}_{j} \cdot \mathbf{p}}{E+m}\right)\right] .
\end{aligned}
$$

Here $m_{\pi}$ and $m$ are the pion and nucleon mass respectively, $f_{\pi N N}$ is the pion-nucleon coupling constant, $\mathbf{p}$ and $\mathbf{p}^{\prime}$ are the momenta of particle $i$ in the center of mass frame before and after

the interaction, $\mathbf{q}=\mathbf{p}^{\prime}-\mathbf{p}$ is the momentum transfer, $E=\sqrt{m^{2}+p^{2}}$, and $E^{\prime}=\sqrt{m^{2}+p^{\prime 2}}$. The $\mu$-dependent term corresponds to nonlocalities in configuration space; it vanishes on-shell where $E=E^{\prime}$. When used in momentum space to solve for deuteron properties, equation (2.2) is multiplied by the $\pi N N$ form factor $F(q)$ to ensure convergence 


$$
F(q)=\left(\frac{\Lambda_{\pi}^{2}-m_{\pi}^{2}}{\Lambda_{\pi}^{2}+q^{2}}\right)^{2}
$$

In the present work we use the cutoff mass $\Lambda_{\pi}=1.2 \mathrm{GeV}$.

The expression for $\mu=-1$, i.e., $v_{\pi}^{\mu=-1}$ can be easily derived using second-order covariant perturbation theory with a pseudoscalar pion-nucleon coupling

$$
H_{\pi N N}^{\mathrm{PS}}=i G \bar{\psi} \gamma_{5} \tau_{i} \psi \phi_{i}
$$

with $G^{2}=4 m^{2} f_{\pi N N}^{2} / m_{\pi}^{2}$. When applying the same technique to the pseudovector-coupling

$$
H_{\pi N N}^{\mathrm{PV}}=-\frac{f_{\pi N N}}{m_{\pi}} \bar{\psi} \gamma^{\mu} \gamma_{5} \tau_{i} \psi \partial_{\mu} \phi_{i}
$$

we can not obtain the $\mu=+1$ expression $v_{\pi}^{\mu=+1}$. The reason is that the derivative coupling of the pion field yields a term dependent on the pion energy $q_{0}$, and within second-order diagrams conservation of energy at each vertex requires $q_{0}=0$. The expression corresponding to $q_{0}=0$ has been given in Ref. [1] up to order $p^{2} / m^{2}$, and is different from $v_{\pi}^{\mu=+1}$ of that order. The correct expression for $v_{\pi}^{\mu=+1}$ [Eq. (2.2)] can be obtained from fourth-order calculations [12].

The $\mu=0$ ("minimal nonlocality") corresponds to on-shell relativistic OPEP

$$
v_{\pi}^{\mu=0}\left(\mathbf{p}^{\prime}, \mathbf{p}\right)=-\frac{f_{\pi N N}^{2}}{m_{\pi}^{2}} \frac{\boldsymbol{\sigma}_{i} \cdot \mathbf{q} \boldsymbol{\sigma}_{j} \cdot \mathbf{q} \boldsymbol{\tau}_{i} \cdot \boldsymbol{\tau}_{j}}{m_{\pi}^{2}+q^{2}} \frac{m}{E} \frac{m}{E^{\prime}}
$$

and has been studied in detail in Ref. [6]. In the nonrelativistic limit, $E \approx E^{\prime} \approx m$, and Eq. (2.6) yields nonrelativistic local OPEP used in the three local potentials (Nijmegen II, Reid93 and AV18)

$$
v_{\pi}^{\mathrm{NR}}(\mathbf{q})=-\frac{f_{\pi N N}^{2}}{m_{\pi}^{2}} \frac{\boldsymbol{\sigma}_{i} \cdot \mathbf{q} \boldsymbol{\sigma}_{j} \cdot \mathbf{q} \boldsymbol{\tau}_{i} \cdot \boldsymbol{\tau}_{j}}{m_{\pi}^{2}+q^{2}}
$$

When Fourier transformed to configuration space, $v_{\pi}^{\mathrm{NR}}[$ Eq. (2.7)] becomes the familiar sum of the tensor and spin-spin interaction terms. The $\delta$-function singularity present in the spin-spin term is usually removed or "smeared" by various form factors, e.g., exponential form factor in Nijmegen II and AV18, dipole form factor in Reid93 and CD-Bonn [Eq. (2.3)]. 
We are interested in understanding the difference between the local and nonlocal potentials. It is sufficient to consider only one of the three available representations of a local potential, which we choose to be AV18. The results can probably be generalized to all modern local potentials.

The OPEP in AV18 is given by the following expression in configuration space

$$
v_{\pi}(\mathbf{r})=\frac{1}{3} m_{\pi} \frac{f_{\pi N N}^{2}}{4 \pi}\left[Y_{\pi}(r) \boldsymbol{\sigma}_{i} \cdot \boldsymbol{\sigma}_{j}+T_{\pi}(r) S_{i j}\right] \boldsymbol{\tau}_{i} \cdot \boldsymbol{\tau}_{j},
$$

where

$$
\begin{aligned}
Y_{\pi}(r) & =\frac{e^{-m_{\pi} r}}{m_{\pi} r}\left(1-e^{-c r^{2}}\right), \\
T_{\pi}(r) & =\left(1+\frac{3}{m_{\pi} r}+\frac{3}{\left(m_{\pi} r\right)^{2}}\right)\left(1-e^{-c r^{2}}\right)^{2}, \\
S_{i j} & =3 \boldsymbol{\sigma}_{i} \cdot \hat{\mathbf{r}} \boldsymbol{\sigma}_{j} \cdot \hat{\mathbf{r}}-\boldsymbol{\sigma}_{i} \cdot \boldsymbol{\sigma}_{j} .
\end{aligned}
$$

The rest of the AV18 is phenomenological and uses the Woods-Saxon and $T_{\pi}^{2}$ functions. When projected onto spin $S=1$ and isospin $T=0$ channel, the overall potential is expressed as

$$
v_{S=1, T=0}(r)=v_{c}(r)+v_{t}(r) S_{i j}+v_{l s}(r) \mathbf{L} \cdot \mathbf{S}+v_{l 2}(r) L^{2}+v_{l s 2}(r)(\mathbf{L} \cdot \mathbf{S})^{2},
$$

where the five terms are called the central, tensor, spin-orbit, quadratic orbital angular momentum and quadratic spin-orbit terms, respectively. The OPEP is obviously included in the central term and is the main contribution to the tensor term.

It is interesting to study deuteron properties using the isoscalar part of AV18 but replace its OPEP with $v_{\pi}^{\mu=-1,0,+1}$ (for future reference, these potentials are denoted as $v_{18}^{\mu=-1,0,+1}$ ). We construct the relativistic Hamiltonian containing $v_{18}^{\mu}$ and relativistic kinetic energy to be phase equivalent to the nonrelativistic Hamiltonian containing the isoscalar part of AV18 and nonrelativistic kinetic energy. The short-range part of the potential $v_{R}$ depends on $\mu$ implicitly in order to reproduce the phase shifts. We adjust the fifteen free parameters in $v_{R}$ and fit the phase shifts in $S=1, T=0$ channel, i.e., ${ }^{3} S_{1}, E_{1},{ }^{3} D_{1},{ }^{3} D_{2},{ }^{3} D_{3}, E_{3},{ }^{3} G_{3}$, and the deuteron binding energy with great accuracy. The deuteron properties are listed in Table I 
and the deuteron wave functions are plotted in Fig. 11. The first row of Table I shows the experimental results; it is followed by results obtained with five modern potentials, and the last three rows are the results obtained with $v_{18}^{\mu=-1,0,+1}$.

The $v_{18}^{\mu=0}$ has been discussed in detail in Ref. [6]. It produces very similar deuteron $D$ state probability $\left(P_{D}=5.73 \%\right)$ to that of the nonrelativistic AV18 (5.76\%). Their deuteron wave functions are also similar as shown in Fig. 11. The $v_{18}^{\mu=0}$ binds the triton slightly more $(\sim 0.1 \mathrm{MeV})$ than AV18 [6]. These similarities between $v_{18}^{\mu=0}$ and AV18 seem reasonable because the Hamiltonian is constrained such that the nonlocalities from the $v_{18}^{\mu=0}$ and relativistic kinetic energy $T_{R}$ have to cancel exactly in the deuteron to give the same binding energy. The small variations in $P_{D}$ and triton binding energy are due to the almost perfect cancellation between $v_{18}^{\mu=0}$ and $T_{R}$ nonlocalities.

In the cases of $\mu=-1$ and +1 , there does not seem to have such a perfect cancellation. The deuteron $P_{D}$ predicted by $v_{18}^{\mu=-1}$ is $4.98 \%$, noticeably smaller than that of $v_{18}^{\mu=0}(5.73 \%)$, while the $P_{D}$ of $v_{18}^{\mu=+1}$ is $6.26 \%$, noticeably larger than that of $v_{18}^{\mu=0}$. This is entirely due to the off-shell terms in $v_{\pi}^{\mu=-1}$ and $v_{\pi}^{\mu=+1}$ absent in $v_{\pi}^{\mu=0}$, because $v_{18}^{\mu=-1,0,+1}$ all have the same short-range parameterization of AV18; only the values of parameters are slightly different in order to fit the data. The $P_{D}$ of $v_{18}^{\mu=-1}(4.98 \%)$ is very close to that of CD-Bonn $(4.83 \%)$ as expected since both potentials have the same OPEP $v_{\pi}^{\mu=-1}$ apart from different pion cut-off mass used in the form factor [Eq. (2.3)]. We use $1.2 \mathrm{GeV}$ for $\Lambda_{\pi}$ while CD-Bonn uses $1.7 \mathrm{GeV}$. When we use $\Lambda_{\pi}=1.7 \mathrm{GeV}$ in our $v_{\pi}^{\mu=-1}$ as a test and refit the phase shifts, the deuteron properties remain almost the same $\left(P_{D}=4.97 \%, D / S\right.$ ratio $=0.0240, Q_{d}=0.0267$ $\left.\mathrm{fm}^{2}\right)$. Therefore the differences between $v_{18}^{\mu=-1}$ and CD-Bonn must come from those in the short-range parts. This is further confirmed by the similar $D$-state wave function at long range $(r>1.5 \mathrm{fm})$. The difference in the deuteron quadrupole moment given by the two potentials is $\sim 1.5 \%$. It is smaller than the $3 \%$ difference in $P_{D}$. This can be understood from the following arguments. In the impulse approximation, deuteron $Q_{d}$ is given by

$$
Q_{d}=\frac{1}{\sqrt{50}} \int_{0}^{\infty}\left(u(r) w(r)-w^{2}(r) / \sqrt{8}\right) r^{2} d r
$$


and it is an "outside" quantity whose major contributions come from the longer-range part of the wave functions which is fairly model-independent. On the other hand, the $D$-state probability $P_{D}$ defined as

$$
P_{D}=\int_{0}^{\infty} w^{2}(r) d r
$$

is an "inside" quantity lacking the extra factor $r^{2}$ in the integrand. Therefore $P_{D}$ is sensitive to the interior region of the wave functions where larger differences exist among various potential models.

Figure 11 also shows another interesting point. The AV18 wave functions are in-between those of $v_{18}^{\mu=+1}$ and $v_{18}^{\mu=0}$. The $v_{18}^{\mu=-1}$ wave functions are farther away, and those of CD-Bonn are the farthest. The difference between the wave functions of AV18 and $v_{18}^{\mu=+1}$ favored by CPT is rather small. This can be easily understood from the following first-order perturbative estimates. The largest contributions of OPEP come from coupling states with small $p$ to large $p^{\prime}$. Therefore we consider the extreme case where $p=0, \mathbf{p}^{\prime}=\mathbf{q}$, and expand Eq. (2.2) in powers of $q^{2} / m^{2}$. This gives

$$
\begin{aligned}
& v_{\pi}^{\mu=-1}(\mathbf{q}, 0)=v_{\pi}^{\mathrm{NR}}\left(1-\frac{3 q^{2}}{4 m^{2}}+\cdots\right), \\
& v_{\pi}^{\mu=0}(\mathbf{q}, 0)=v_{\pi}^{\mathrm{NR}}\left(1-\frac{q^{2}}{2 m^{2}}+\cdots\right), \\
& v_{\pi}^{\mu=+1}(\mathbf{q}, 0)=v_{\pi}^{\mathrm{NR}}\left(1-\frac{q^{2}}{4 m^{2}}+\cdots\right) .
\end{aligned}
$$

If we treat OPEP as a perturbative term in the Hamiltonian, the first order correction to the amplitude of a state with large momentum $\mathbf{q}$ is given by

$$
\psi(\mathbf{q}) \approx \frac{v_{\pi}(\mathbf{q}, 0)}{E(q)-E(0)} \psi(\mathbf{q}=0)
$$

Here $v_{\pi}$ can be $v_{\pi}^{\mathrm{NR}}$ or $v_{\pi}^{\mu}$ depending on whether the Hamiltonian is nonrelativistic or relativistic. In the nonrelativistic case, $E(q)-E(0) \approx q^{2} / m$, while in the relativistic case, $E(q)-E(0) \approx q^{2} / m \times\left(1-q^{2} / 4 m^{2}\right)$. Therefore the difference between the first order, large q, amplitudes of $v_{\pi}^{\mu}$ and $v_{\pi}^{\mathrm{NR}}$ is given by 


$$
\begin{aligned}
\psi^{\mu=-1}(\mathbf{q})-\psi^{\mathrm{NR}}(\mathbf{q}) & \approx-\frac{q^{2}}{2 m^{2}} \psi^{\mathrm{NR}}(\mathbf{q}) \\
\psi^{\mu=0}(\mathbf{q})-\psi^{\mathrm{NR}}(\mathbf{q}) & \approx-\frac{q^{2}}{4 m^{2}} \psi^{\mathrm{NR}}(\mathbf{q}) \\
\psi^{\mu=+1}(\mathbf{q})-\psi^{\mathrm{NR}}(\mathbf{q}) & \approx 0 .
\end{aligned}
$$

This estimate suggests that the $v_{\pi}^{\mu=+1}$ wave function is the closest to the nonrelativistic wave function, that of $v_{\pi}^{\mu=0}$ is the second closest, and that of $v_{\pi}^{\mu=-1}$ is the farthest. In reality, other components of $v\left(\mathbf{p}^{\prime}, \mathbf{p}\right)$ contribute as well, and the OPEP can not be treated perturbatively. The exact calculations show (Fig. 1) that the nonrelativistic wave function is in-between those of $v_{18}^{\mu=+1}$ and $v_{18}^{\mu=0}$. This implies that the wave functions obtained from nonrelativistic local potentials such as AV18 and nonrelativistic kinetic energy provide excellent approximation to those with $v_{18}^{\mu=+1}$ and $v_{18}^{\mu=0}$ and relativistic kinetic energy.

The expectation values of the kinetic energy $T$, OPEP $v_{\pi}$ and the phenomenological short-range part $v_{R}$ of various potentials are compared in Table II. As we can see, the individual terms change slightly among various potentials, but the sum of them gives identical total energy of $-2.242 \mathrm{MeV}$ by construction. The experimental value of deuteron binding energy $(-2.224 \mathrm{MeV})$ is obtained from the full AV18 with electromagnetic interactions. From Table II, it appears that the individual terms of $v_{18}^{\mu=+1}$ are the closest to those of AV18, those of $v_{18}^{\mu=0}$ are the second closest, and those of $v_{18}^{\mu=-1}$ are the farthest. Also listed in this table are the on-shell $\left(v_{\pi}^{\text {on-shell }}\right)$ and off-shell $\left(v_{\pi}^{\text {off-shell }}\right)$ contributions to the OPEP. The sum of the two terms gives the total $v_{\pi}$ [Eq. (2.2)]. For AV18 and $v_{18}^{\mu=0}$, the off-shell contributions are obviously zero, and the on-shell contributions are the nonrelativistic OPEP [Eq. (2.8)] and on-shell relativistic OPEP [Eq. (2.6)], respectively. For $v_{18}^{\mu= \pm 1}$, the off-shell contribution is much smaller than the on-shell one because the former is of order $p^{2} / m^{2}$ of the latter in the leading order.

The deuteron charge form factors $F_{c}^{2}$ and $T_{20}$ calculated in impulse approximation are plotted in Figs. 2 and 3 . Notice that the results predicted by AV18 are in-between those of $v_{18}^{\mu=+1}$ and $v_{18}^{\mu=0}$. The difference between the $T_{20}$ obtained with CD-Bonn and AV18 is much larger than that calculated with $v_{18}^{\mu}$ of various choices of $\mu$ implying that the nonlocality in 
the shorter-range part of CD-Bonn is the main cause.

\section{UNITARY EQUIVALENCE}

Friar [7,8] suggested two decades ago that the physics of pseudovector and pseudoscalar couplings of pions to nucleons is related via the equivalence theorem, originating from the Dyson transformation [13]. Basically, the theorem states [8] that the one-pion-exchange currents, together with matrix elements of the impulse charge operator calculated with a potential including OPEP, must be the same for both PS and PV couplings to order $G^{2}$. As an interesting byproduct of Friar's work, the unitary transformation involved can change the deuteron $D$-state probability, indicating that this quantity is not measurable [14].

It is interesting to test this theorem using our relativistic Hamiltonian [Eq. (2.1)] for $\mu=-1,0,+1$. The Hamiltonian also contains a short-range potential $v_{R}$ which is implicitly dependent on $\mu$ in order to fit the data. We can rewrite Eq. (2.1) as the following

$$
H_{\mathrm{exact}}^{\mu}=T_{R}+v_{\pi}^{\mu=0}+\Delta v_{\pi}^{\mu}+v_{R}^{\mu}
$$

where $T_{R}=2 \sqrt{p^{2}+m^{2}}-2 m$ is the relativistic kinetic energy, $v_{\pi}^{\mu=0}$ is the on-shell relativistic OPEP given by Eq. (2.6) and $\Delta v_{\pi}^{\mu}$ is the off-shell term in Eq. (2.2) given by

$$
\Delta v_{\pi}^{\mu}\left(\mathbf{p}^{\prime}, \mathbf{p}\right)=-\mu \times \frac{f_{\pi N N}^{2}}{m_{\pi}^{2}} \frac{\boldsymbol{\tau}_{i} \cdot \boldsymbol{\tau}_{j}}{m_{\pi}^{2}+q^{2}} \frac{m}{E} \frac{m}{E^{\prime}}\left(E^{\prime}-E\right)\left(\frac{\boldsymbol{\sigma}_{i} \cdot \mathbf{p}^{\prime} \boldsymbol{\sigma}_{j} \cdot \mathbf{p}^{\prime}}{E^{\prime}+m}-\frac{\boldsymbol{\sigma}_{i} \cdot \mathbf{p} \boldsymbol{\sigma}_{j} \cdot \mathbf{p}}{E+m}\right) .
$$

In light nuclei, the small binding energy comes from the large cancellation between the kinetic energy and two-body potential. Hence $v_{\pi}^{\mu=0}$ and $v_{R}^{\mu}$ in Eq. (3.1) are of the same order of magnitude as $T_{R}$. The off-shell term $\Delta v_{\pi}^{\mu}$ is much smaller, its leading term is of order $p^{2} / m^{2}$ of $v_{\pi}^{\mu=0}$. It seems that in deuteron the off-shell term $\Delta v_{\pi}$ is comparable to $v_{R}$ as shown in Table II. This is because deuteron is a loosely bound state with a very large rms radius, thus the OPEP accounts for more than $95 \%$ of the two-body potential, yielding a very small $v_{R}$. In light nuclei with $A>2$, the OPEP still accounts for a large portion of the two-body potential $(>70 \%)$, but $v_{R}$ is about the same order of magnitude as the kinetic energy and OPEP 15 and $\Delta v_{\pi}^{\mu}$ is probably much smaller than $v_{R}$. 
The Hamiltonian [Eq. (3.1)] can also be expressed, up to order $p^{2} / m^{2}$, as the following unitary transformation

$$
H_{\mathrm{uni}}^{\mu}=e^{-i U} H_{0} e^{i U} \approx H_{0}+i\left[H_{0}, U\right]
$$

where $H_{0}$ can be conveniently chosen as

$$
H_{0}=H_{\text {exact }}^{\mu=0}=T_{R}+v_{\pi}^{\mu=0}+v_{R}^{\mu=0} .
$$

The unitary operator which satisfies Eq. (3.3) is found to be

$$
i U=-\mu \times \frac{1}{2} \frac{f_{\pi N N}^{2}}{m_{\pi}^{2}} \frac{\boldsymbol{\tau}_{i} \cdot \boldsymbol{\tau}_{j}}{m_{\pi}^{2}+q^{2}} \frac{m}{E} \frac{m}{E^{\prime}}\left(\frac{\boldsymbol{\sigma}_{i} \cdot \mathbf{p}^{\prime} \boldsymbol{\sigma}_{j} \cdot \mathbf{p}^{\prime}}{E^{\prime}+m}-\frac{\boldsymbol{\sigma}_{i} \cdot \mathbf{p} \boldsymbol{\sigma}_{j} \cdot \mathbf{p}}{E+m}\right) .
$$

It can be easily verified that the commutator

$$
\left[T_{R}, i U\right]=\Delta v_{\pi}^{\mu}
$$

The wave function is transformed, consistently with the Hamiltonian, in the following way

$$
\left|\psi^{\mu}\right\rangle^{\text {uni }}=e^{-i U}\left|\psi_{0}\right\rangle \approx(1-i U)\left|\psi_{0}\right\rangle
$$

where $\left|\psi_{0}\right\rangle$ is the wave function of $H_{0}$ obtained by solving Schrödinger equation. The methods used to solve Eq. (3.7) for the deuteron are given in the Appendix.

Earlier works by Friar [5,7], Desplanques and Amghar [16] and Adam et al. [1] used a nonrelativistic kinetic energy $p^{2} / m$ in the Hamiltonian, and kept the leading order $\left(p^{2} / m^{2}\right)$ terms in the "relativistic" OPEP and the unitary operator. This seems to be inconsistent with the relativistic considerations, nor is it computationally easier than the exact treatment since all the calculations involving the OPEP off-shell term are performed in momentum space. In this work we use a relativistic kinetic energy and keep the relativistic OPEP and unitary operator $i U$ in their exact forms.

From Eqs. (3.3), (3.4) and (3.5), we find that the unitarily transformed Hamiltonian is not exactly the same as the $H_{\text {exact }}^{\mu}$

$$
H_{\mathrm{uni}}^{\mu}=H_{\text {exact }}^{\mu}+\Delta H^{\mu}
$$


The correction term is

$$
\Delta H^{\mu}=v_{R}^{\mu=0}+\left[v_{\pi}^{\mu=0}, i U\right]+\left[v_{R}^{\mu=0}, i U\right]-v_{R}^{\mu}
$$

The two commutators $\left[v_{\pi}^{\mu=0}, i U\right]$ and $\left[v_{R}^{\mu=0}, i U\right]$ are of the same order as $\Delta v_{\pi}^{\mu}$, but the first commutator has the range of two-pion exchange interaction while the second one has even shorter range. Both can be absorbed into the phenomenological short-range part $v_{R}^{\mu=0}$ to obtain $v_{R}^{\mu \prime}$. If this $v_{R}^{\mu \prime}$ is exactly the same as $v_{R}^{\mu}$, then $H_{\text {uni }}^{\mu}=H_{\text {exact }}^{\mu}$, and $H^{\mu}$ is unitarily equivalent to $H^{\mu=0}$. However, it is unlikely that $v_{R}^{\mu \prime}=v_{R}^{\mu}$ as demonstrated in the wave functions shown in Fig. 4 and deuteron properties listed in Table III.

As we can see in Fig. \#, the unitarily transformed deuteron wave functions are slightly different from the exact wave functions obtained by solving the Schrödinger equation, mostly at short range. The unitary transformation works rather well for the $D$-state. It also works well for the $S$-state at long range but somewhat breaks down at very short range $(r<0.3 \mathrm{fm})$. This can be understood from the following arguments. The unitary transformation discussed in this work is related to OPEP. The long-range parts of the wave functions are mostly determined by the OPEP, thus they are related very well by the unitary transformation. The $D$-state is most sensitive to the tensor force whose main contribution comes from the OPEP. The $S$-state, however, is sensitive to both central and tensor parts of the potential at short range, thus the unitary transformation starts to deviate from the exact $S$-state wave function at $r<0.3 \mathrm{fm}$. This deviation at such short range may have very small impact on the observables. For example, the deuteron binding energies calculated with the unitarily transformed wave functions for $\mu=-1$ and +1 are reduced by only $\sim 8 \%$ as compared with the exact result, indicating that the unitary transformation is a good approximation to the exact solution.

The deuteron properties obtained with unitary transformation are compared with those from solving Schrödinger equation in Table III. The differences between the exact solutions and the results of unitary transformation are obviously caused by the short-range part of the potential in $\Delta H$. These differences are rather small indicating that the potentials $v_{18}^{\mu}$ 
for $\mu=-1,0$ and +1 are, to large extent, unitarily equivalent. Desplanques and Amghar [16] studied unitary equivalence between Paris $(\mu=0)$ and the older Bonn $(\mu=-1)$ potentials before the birth of the modern potentials. The differences between the results of Bonn and the unitarily transformed Paris, or Paris and unitarily transformed Bonn seem to be larger than the present work, indicating that the Paris and Bonn potentials are not as unitarily equivalent as our $v_{18}^{\mu}$ models. The reason is probably due to the rather different structures in the short-range part of the two potentials, and possibly due to using the leading order terms in kinetic energy, OPEP and unitary operator instead of their exact expressions. Adam et al. [11] did a similar study as the present work, except that they used a pure OPEP model to describe the two-nucleon system, and they also used leading order expressions for kinetic energy, OPEP and unitary operator. They studied deuteron properties for various values of $\mu$ and $\nu$. Their deuteron $P_{D}$ and $Q_{d}$ for $\nu=1 / 2$ (no retardation) and $\mu=-1,0,+1$ seem to be larger than ours, probably because they used a pure OPEP model instead of a realistic model which fits $N N$ phase shifts as well as deuteron binding energy. However, the amount of changes from before and after unitary transformation are similar to ours.

The deuteron $P_{D}$ of current work is plotted in Fig. 5. The $P_{D}$ obtained with unitary transformation seems to be linear in $\mu$, while the exact results are slightly nonlinear. This can be easily understood from the expression of the unitarily transformed wave functions given in Eq. (A16). We can rewrite the wave functions as

$$
\begin{aligned}
& R_{0}^{\text {uni }}(p)=R_{0}(p)+\mu \times \delta R_{0}(p), \\
& R_{2}^{\text {uni }}(p)=R_{2}(p)+\mu \times \delta R_{2}(p),
\end{aligned}
$$

where $R_{0}(p)$ and $R_{2}(p)$ are the normalized $S$ - and $D$-state radial wave functions, $\delta R_{0}(p)$ and $\delta R_{2}(p)$ are the second terms in Eq. (A16). In the dominant region of the wave functions, i.e., $p<5 \mathrm{fm}^{-1}, R_{0}(p) \gg \delta R_{0}(p), R_{2}(p) \gg \delta R_{2}(p)$. The deuteron $D$-state probability is given by

$$
\begin{aligned}
P_{D}^{\text {uni }}=P_{D}^{0} & +\mu \times\left[-2 P_{D}^{0} \int_{0}^{\infty} R_{0}(p) \delta R_{0}(p) p^{2} d p+2\left(1-P_{D}^{0}\right) \int_{0}^{\infty} R_{2}(p) \delta R_{2}(p) p^{2} d p\right] \\
& + \text { terms of order } \mu^{2} \text { and higher, }
\end{aligned}
$$


where $P_{D}^{0}$ is the deuteron $D$-state probability of $\mu=0$. The terms of order $\mu^{2}$ and higher are negligibly small and can be neglected, therefore $P_{D}$ obtained from the unitary transformation is approximately linear to $\mu$. The exact $P_{D}$ is slightly nonlinear reflecting the difference of the short-range part of the potentials.

\section{LOCAL POTENTIALS}

Before we draw any conclusions, let's ask a question: is the small $P_{D}$ a unique feature from $v_{\pi}^{\mu=-1}$, or the large $P_{D}$ a unique feature from $v_{\pi}^{\mu=+1}$ ? In other words, can we find a local potential which fits experimental data and still gives $P_{D}<5 \%$ or $P_{D}>6 \%$ ?

To explore such a possibility, we attempt to lower the $P_{D}$ to $\sim 5 \%$ by using AV18 as trial local potential in our Hamiltonian, together with a nonrelativistic kinetic energy. There are fifteen free parameters in the AV18 representation [Eq. (2.12)]. Just by varying these parameters to fit the data and simultaneously constraining $P_{D} \approx 5 \%$ can result in good fits to ${ }^{3} S_{1},{ }^{3} D_{1},{ }^{3} D_{2},{ }^{3} D_{3}, E_{3}$ and ${ }^{3} G_{3}$ in addition to deuteron binding energy, but not to the mixing angle $E_{1}$. At higher energies (200-400 MeV) $E_{1}$ comes out significantly below the Nijmegen values.

Since $P_{D}$ is sensitive to the tensor force, in order to get smaller $P_{D}$, we need to reduce the tensor force. We have tried various approaches summarized below: (i) Multiply the entire tensor force $v_{t}(r)$ by a factor $\left(1-e^{-c_{1} r^{2}}\right)$, and adjust the parameter $c_{1}$ in addition to the fifteen parameters; (ii) Add a factor $c_{2} r\left(1-e^{-c_{3} r^{2}}\right)$ to the tensor force, and vary the parameters $c_{2}$ and $c_{3}$ in addition to the fifteen parameters; (iii) Use the wave functions obtained with $v_{18}^{\mu=-1}$ whose $P_{D}$ is $4.98 \%$ and solve the Schrödinger equation for local $v_{c}$ and $v_{t}$ (denote them as "local $\mu=-1$ "), keeping $v_{l s}, v_{l 2}$ and $v_{l s 2}$ fixed. These potentials are compared with those in the AV18 in Fig. 6. As we can see, the "local $\mu=-1$ " $v_{c}$ and $v_{t}$ are both reduced at the short-range to reproduce the deuteron binding energy and obtain a smaller $P_{D}$. We then multiply or add various functions to $v_{c}$ and $v_{t}$ to reproduce this effect. None of these approaches yield a good $\chi^{2}$ fit. The largest discrepancies are for $E_{1}$. 
This is not surprising because $E_{1}$ is most sensitive to the tensor force, unlike any other states. When restricting $P_{D}$ to be $\sim 5 \%$, we are reducing the tensor force which in turn reduces the $E_{1}$. Other states can make trade-offs among $v_{c}, v_{l s}, \cdots$. In contrast, when the OPEP tensor force is modified by the off-shell term in Eq. (2.2), only the off-diagonal elements $v\left(p, p^{\prime}\right)$ is reduced, but the Born term $v(p, p)$ remains the same. This seems to suggest two things: (i) the tensor Born term plays an important role in fitting to $E_{1}$ phase shift; (ii) weakening the tensor force off-diagonally does not seem to affect $E_{1}$, however it produces a smaller $P_{D}$ of the deuteron.

In summary, we couldn't find a local potential which gives a deuteron $P_{D}$ as small as $5 \%$. We can probably extrapolate this statement to $P_{D}$ as large as $6 \%$. Therefore, it seems that the different deuteron $P_{D}$ 's are associated with the various off-shell behaviors of the OPEP.

\section{CONCLUSIONS}

We studied deuteron properties with potentials containing $\mu=-1,0$, and +1 OPEP offshell behaviors. We tested the unitary equivalence among these potentials by comparing to the exact solutions of Schrödinger equation, and we found the following interesting results:

(i) The off-shell term involving the coupling of nucleon spin and momentum is the primary cause for the smaller deuteron $P_{D}$ for CD-Bonn and $v_{18}^{\mu=-1}$, and consequently a higher triton binding energy. We also found that it is not possible to get a deuteron with $P_{D}$ as small as $5 \%$ with the local OPEP [Eq. (2.8)].

(ii) The deuteron wave functions, charge form factors and $T_{20}$ for $\mu=+1$ favored by CPT are very close to those of the nonrelativistic AV18.

(iii) The $v_{18}^{\mu=-1,0,+1}$ models are, to large extent, unitarily equivalent.

(iv) The differences due to unitary transformations related to OPEP are smaller than the difference between AV18 and CD-Bonn potential.

The charge form factors and $T_{20}$ shown in this work are calculated in impulse approximation. After including the meson-exchange current operator obtained with unitary trans- 
formation consistent with the Hamiltonians and wave functions, the deuteron $A$ and $B$ structure functions as well as $T_{20}$ turn out to be rather similar for $v_{18}^{\mu=-1,0,+1}$ and AV18, but they differ significantly from those of CD-Bonn [17]. This suggests that AV18 and CD-Bonn are not well-related by the kind of unitary transformation discussed in this work, i.e., the one that deals with OPEP.

In summary, we find that a nonrelativistic Hamiltonian containing local potentials (AV18, Nijmegen II, Reid93) and nonrelativistic kinetic energy provides an excellent approximation to a relativistic Hamiltonian containing a relativistic OPEP of pseudovector pion-nucleon coupling and relativistic kinetic energy, in predicting properties of nuclei. This may matter little for two-nucleon systems such as the deuteron discussed in this work, or three-nucleon systems such as the triton discussed in Refs. [3, 18] for which momentum space as well as configuration space computational methods can be applied. However, when we go up to nuclei having $A>3$, momentum-space techniques face great computational difficulties, leaving the configuration-space calculations as the main approach for high-accuracy computations [15,19], in which case a local nonrelativistic Hamiltonian is to be preferred.

\section{ACKNOWLEDGMENTS}

I wish to thank V. R. Pandharipande and R. Schiavilla for their valuable input and many contributions to this subject. I have benefited tremendously from many inspirational conversations with them. I also thank A. Arriaga, J. L. Friar, and R. Machleidt for many interesting discussions. The calculations were made possible by generous grants of time on the National Energy Research Supercomputer Center. The support of the U. S. Department of Energy is gratefully acknowledged. 


\section{APPENDIX A: UNITARY TRANSFORMATION OF THE DEUTERON WAVE FUNCTIONS}

Consider the case of deuteron. The Hamiltonian is given by Eq. (3.4) for $\mu=0$. The wave function in momentum space is expressed as

$$
\left|\psi_{0}(\mathbf{p})\right\rangle=R_{0}(p) \mathcal{Y}_{110}(\hat{\mathbf{p}})+R_{2}(p) \mathcal{Y}_{112}(\hat{\mathbf{p}})
$$

where $R_{0}(p)$ and $R_{2}(p)$ are the $S$ - and $D$-state radial wave functions, $\mathcal{Y}_{L S J}(\hat{\mathbf{p}})$ are the spinangle functions

$$
\mathcal{Y}_{L S J}=(-i)^{L} \sum_{M_{S}}\left\langle L, M_{L}=M_{J}-M_{S}, S, M_{S} \mid J, M_{J}\right\rangle Y_{L, M_{L}}\left|S, M_{S}\right\rangle
$$

Here $J, S, L$ are the total, spin, and orbital angular momentum, respectively, and $M_{J}, M_{S}, M_{L}$ are their projections along z-axis. From Eq. (3.7), the wave functions of $\mu= \pm 1$ are given by

$$
\left|\psi(\mathbf{p})^{\mu= \pm 1}\right\rangle^{\text {uni }}=\left|\psi_{0}(\mathbf{p})\right\rangle-\int \frac{d^{3} p}{(2 \pi)^{3}} i U\left(\mathbf{p}, \mathbf{p}^{\prime}\right)\left|\psi_{0}\left(\mathbf{p}^{\prime}\right)\right\rangle
$$

where the unitary operator is given by Eq. (3.5) multiplied by the form factor [Eq. (2.3)]. We can rewrite

$$
\frac{1}{m_{\pi}^{2}+q^{2}}\left(\frac{\Lambda_{\pi}^{2}-m_{\pi}^{2}}{\Lambda_{\pi}^{2}+q^{2}}\right)^{2}=\frac{1}{m_{\pi}^{2}+q^{2}}-\frac{1}{\Lambda_{\pi}^{2}+q^{2}}+\left(\Lambda_{\pi}^{2}-m_{\pi}^{2}\right) \frac{d}{d \Lambda_{\pi}^{2}} \frac{1}{\Lambda_{\pi}^{2}+q^{2}}
$$

Then use

$$
\frac{1}{m_{\pi}^{2}+q^{2}}=\frac{2 \pi}{p p^{\prime}} \sum_{l m} Q_{l}\left(z_{m_{\pi}}\right) Y_{l m}^{*}(\hat{\mathbf{p}}) Y_{l m}\left(\hat{\mathbf{p}}^{\prime}\right)
$$

where $Q_{l}\left(z_{m_{\pi}}\right)$ is the Legendre function of the second kind and

$$
z_{m_{\pi}}=\frac{p^{2}+p^{2}+m_{\pi}^{2}}{2 p p^{\prime}}
$$

Because deuteron has only $l=0$ and 2 states, we will only need $Q_{0}$ and $Q_{2}$

$$
\begin{aligned}
& Q_{0}(z)=\frac{1}{2} \ln \left(\frac{z+1}{z-1}\right) \\
& Q_{2}(z)=\frac{1}{2}\left(3 z^{2}-1\right) Q_{0}(z)-\frac{3}{2} z
\end{aligned}
$$


Using these, Eq. (A4) becomes

$$
\frac{1}{m_{\pi}^{2}+q^{2}}\left(\frac{\Lambda_{\pi}^{2}-m_{\pi}^{2}}{\Lambda_{\pi}^{2}+q^{2}}\right)^{2}=\frac{2 \pi}{p p^{\prime}} \sum_{l m} \bar{Q}_{l}\left(p^{\prime}, p\right) Y_{l m}^{*}(\hat{\mathbf{p}}) Y_{l m}\left(\hat{\mathbf{p}}^{\prime}\right)
$$

where

$$
\bar{Q}_{l}\left(p^{\prime}, p\right)=Q_{l}\left(z_{m_{\pi}}\right)-Q_{l}\left(z_{\Lambda_{\pi}}\right)+\left(\Lambda_{\pi}^{2}-m_{\pi}^{2}\right) \frac{d}{d \Lambda_{\pi}^{2}} Q_{l}\left(z_{\Lambda_{\pi}}\right)
$$

Then we use the following relations

$$
\begin{aligned}
\boldsymbol{\sigma}_{1} \cdot \mathbf{p} \boldsymbol{\sigma}_{2} \cdot \mathbf{p} & =\frac{1}{3} p^{2}\left(S_{12}(\hat{\mathbf{p}})+\boldsymbol{\sigma}_{1} \cdot \boldsymbol{\sigma}_{2}\right) \\
S_{12}(\hat{\mathbf{p}}) \mathcal{Y}_{110}(\hat{\mathbf{p}}) & =\sqrt{8} \mathcal{Y}_{112}(\hat{\mathbf{p}}) \\
S_{12}(\hat{\mathbf{p}}) \mathcal{Y}_{112}(\hat{\mathbf{p}}) & =\sqrt{8} \mathcal{Y}_{110}(\hat{\mathbf{p}})-2 \mathcal{Y}_{112}(\hat{\mathbf{p}}), \\
\boldsymbol{\sigma}_{1} \cdot \boldsymbol{\sigma}_{2} \mathcal{Y}_{110}(\hat{\mathbf{p}}) & =\mathcal{Y}_{110}(\hat{\mathbf{p}}) \\
\boldsymbol{\sigma}_{1} \cdot \boldsymbol{\sigma}_{2} \mathcal{Y}_{112}(\hat{\mathbf{p}}) & =\mathcal{Y}_{112}(\hat{\mathbf{p}})
\end{aligned}
$$

Finally, putting everything together, we get

$$
\left|\psi^{\mu}(\mathbf{p})\right\rangle^{\mathrm{uni}}=R_{0}^{\mathrm{uni}}(p) \mathcal{Y}_{110}(\hat{\mathbf{p}})+R_{2}^{\mathrm{uni}}(p) \mathcal{Y}_{112}(\hat{\mathbf{p}})
$$

where

$$
\begin{aligned}
R_{0}^{\text {uni }}(p)=R_{0}(p) & +\mu \times \frac{1}{8 \pi^{2}} \frac{f_{\pi N N}^{2}}{m_{\pi}^{2}} \frac{m^{2}}{E p} \int \frac{p^{\prime} d p^{\prime}}{E^{\prime}}\left\{\left(E^{\prime}-E\right) \bar{Q}_{0}\left(p^{\prime}, p\right) R_{0}\left(p^{\prime}\right)\right. \\
& \left.+\sqrt{8}\left[\left(E^{\prime}-m\right) \bar{Q}_{0}\left(p^{\prime}, p\right)-(E-m) \bar{Q}_{2}\left(p^{\prime}, p\right)\right] R_{2}\left(p^{\prime}\right)\right\} \\
R_{2}^{\text {uni }}(p)=R_{2}(p) & +\mu \times \frac{1}{8 \pi^{2}} \frac{f_{\pi N N}^{2}}{m_{\pi}^{2}} \frac{m^{2}}{E p} \int \frac{p^{\prime} d p^{\prime}}{E^{\prime}}\left\{\sqrt{8}\left(E^{\prime}-E\right) \bar{Q}_{0}\left(p^{\prime}, p\right) R_{0}\left(p^{\prime}\right)\right. \\
& \left.+\left[\left(E^{\prime}-m\right) \bar{Q}_{0}\left(p^{\prime}, p\right)-(E-m) \bar{Q}_{2}\left(p^{\prime}, p\right)\right] R_{2}\left(p^{\prime}\right)\right\}
\end{aligned}
$$

The wave functions should be normalized to 1, then Fourier transformed to configuration space to obtain the $S$ - and $D$-state wave functions shown in Fig. 4 . 


\section{REFERENCES}

* $\quad$ Electronic address: jforest@jlab.org

[1] V. G. J. Stoks, R. A. M. Klomp, C. P. F. Terheggen, and J. J. de Swart, Phys. Rev. C 49, 2950 (1994).

[2] R. B. Wiringa, V. G. J. Stoks, and R. Schiavilla, Phys. Rev. C 51, 38 (1995).

[3] R. Machleidt, F. Sammarruca, and Y. Song, Phys. Rev. C 53, R1483 (1996).

[4] V. G. J. Stoks, R. A. M. Klomp, M. C. M. Rentmeester, and J. J. de Swart, Phys. Rev. C 48, 792 (1993).

[5] J. L. Friar, LA-UR-99-296, nucl-th/9901082, 1999.

[6] J. L. Forest, V. R. Pandharipande, and A. Arriaga, to be appearing in Phys. Rev. C July version, 1999.

[7] J. L. Friar, Phys. Rev. C 22, 796 (1980).

[8] J. L. Friar, Ann. Phys. (N.Y.) 104, 380 (1977).

[9] S. A. Coon and J. L. Friar, Phys. Rev. C 34, 1060 (1986).

[10] J. L. Forest, V. R. Pandharipande, and J. L. Friar, Phys. Rev. C 52, 568 (1995).

[11] J. Adam, Jr., H. Göller, and H. Arenhövel, Phys. Rev. C 48, 370 (1993).

[12] M. Sugawara and S. Okubo, Phys. Rev. 117, 605 and 611 (1960).

[13] F. J. Dyson, Phys. Rev. 73, 929 (1948).

[14] J. L. Friar, Phys. Rev. C 20, 325 (1979).

[15] B. S. Pudliner, V. R. Pandharipande, J. Carlson, and R. B. Wiringa, Phys. Rev. Lett. 74, 4396 (1995); B. S. Pudliner, V. R. Pandharipande, J. Carlson, Steven C. Pieper, and R. B. Wiringa, Phys. Rev. C 56, 1720 (1997). 
[16] B. Desplanques and A. Amghar, Z. Phys. A344, 191 (1992).

[17] R. Schiavilla (private communication).

[18] J. L. Friar, G. L. Payne, V.G.J. Stoks, and J.J. de Swart, Phys. Lett. B331, 4 (1993).

[19] J. Carlson and R. Schiavilla, Rev. Mod. Phys. 70, 743 (1998). 


\section{FIGURES}

FIG. 1. Deuteron $S$ - and $D$-state wave functions.

FIG. 2. Deuteron charge form factors squared for spin projections $M_{d}=0$ and 1 in impulse approximation, for various potentials.

FIG. 3. Deuteron tensor polarizing power $T_{20}$ in impulse approximation, for various potentials.

FIG. 4. Comparison of deuteron wave functions obtained from unitary transformation and those from solving Schrödinger equation.

FIG. 5. Deuteron $D$-sate probability as a function of $\mu$. The circles represent the $P_{D}$ obtained by solving Schrödinger equation, while the diamond symbols represent those from unitary transformation.

FIG. 6. Comparison of the "local $\mu=-1$ " central and tensor potentials with AV18. 


\section{TABLES}

TABLE I. Deuteron properties predicted by the modern potentials and $v_{18}^{\mu}$ for $\mu=-1,0,+1$.

\begin{tabular}{|c|c|c|c|c|}
\hline & Character & $Q_{d}\left(\mathrm{fm}^{2}\right)$ & $D / S$ ratio & $P_{D}(\%)$ \\
\hline Experiment & nonlocal & $0.2859(3)$ & $0.0256(4)$ & - \\
\hline AV18 & local & 0.270 & 0.0250 & 5.76 \\
\hline Nijmegen II & local & 0.271 & 0.0252 & 5.64 \\
\hline Reid93 & local & 0.270 & 0.0251 & 5.70 \\
\hline Nijmegen I & nonlocal & 0.272 & 0.0253 & 5.66 \\
\hline CD-Bonn $(\mu=-1)$ & nonlocal & 0.270 & 0.0255 & 4.83 \\
\hline$v_{18}^{\mu=-1}$ & nonlocal & 0.266 & 0.0253 & 4.98 \\
\hline$v_{18}^{\mu=0}$ & nonlocal & 0.271 & 0.0258 & 5.73 \\
\hline$v_{18}^{\mu=+1}$ & nonlocal & 0.272 & 0.0260 & 6.26 \\
\hline
\end{tabular}

TABLE II. Expectation values of kinetic energy $T$, OPEP $v_{\pi}$ and the phenomenological short-range part $v_{R}$ of various potentials (in $\mathrm{MeV}$ ).

\begin{tabular}{lrcrr}
\hline \hline & AV18 & $v_{18}^{\mu=-1}$ & $v_{18}^{\mu=0}$ & $v_{18}^{\mu=+1}$ \\
\hline$\langle E\rangle^{\dagger}$ & -2.242 & -2.242 & -2.242 & -2.242 \\
\hline$\langle T\rangle$ & 19.882 & 17.352 & 18.877 & 20.161 \\
$\left\langle v_{\pi}\right\rangle$ & -21.355 & -15.642 & -18.797 & -21.486 \\
$\left\langle v_{R}\right\rangle$ & -0.769 & -3.952 & -2.322 & -0.917 \\
\hline$\left\langle v_{\pi}^{\text {on-shell }}\right\rangle$ & -21.355 & -17.399 & -18.797 & -19.407 \\
$\left\langle v_{\pi}^{\text {off-shell }}\right\rangle$ & 0 & 1.757 & 0 & -2.079 \\
\hline \hline
\end{tabular}

$\dagger$ without electromagnetic interactions 
TABLE III. Deuteron $D$-state probability $P_{D}$, quadrupole moment $Q_{d}$ and asymptotic $D / S$ ratio for various values of $\mu$.

\begin{tabular}{lccr}
\hline \hline$\mu$ & $P_{D}(\%)$ & $Q_{d}\left(\mathrm{fm}^{2}\right)$ & $D / S$ ratio \\
\hline 0 (exact) & 5.73 & 0.271 & 0.0258 \\
-1 (unitary) & 5.16 & 0.268 & 0.0253 \\
-1 (exact) & 4.98 & 0.266 & 0.0253 \\
+1 (unitary) & 6.34 & 0.273 & 0.0263 \\
+1 (exact) & 6.26 & 0.273 & 0.0260 \\
\hline \hline
\end{tabular}




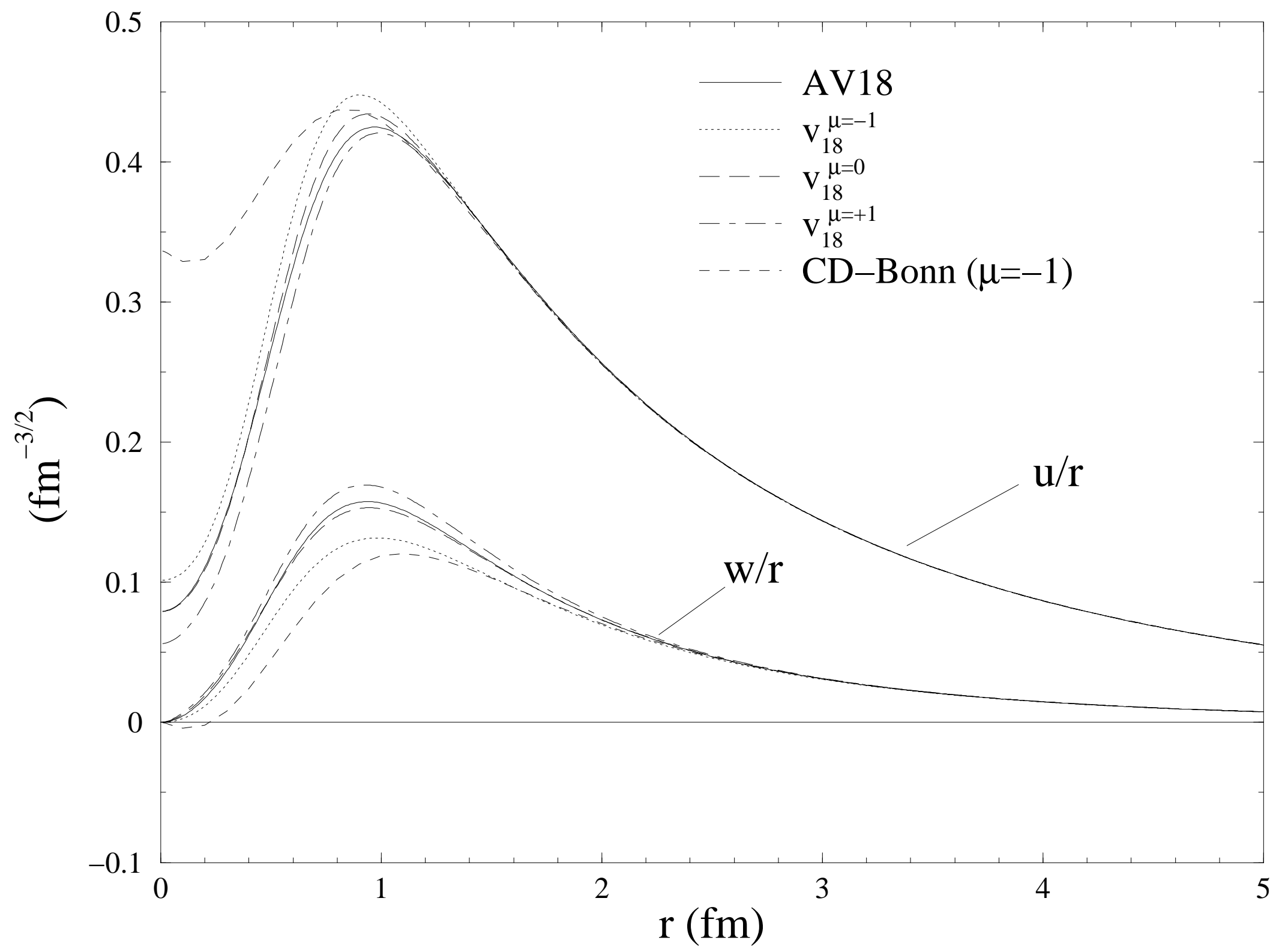




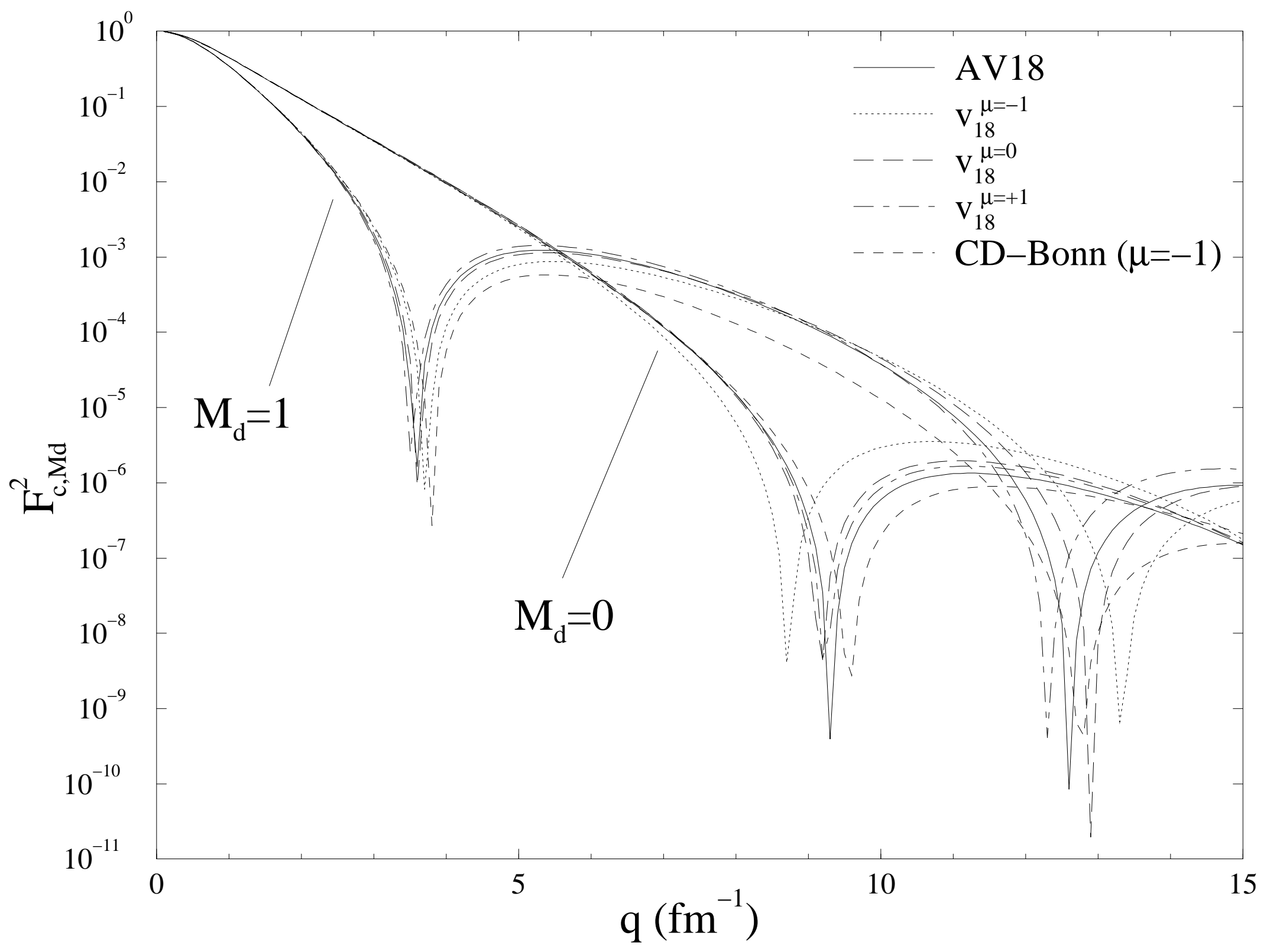




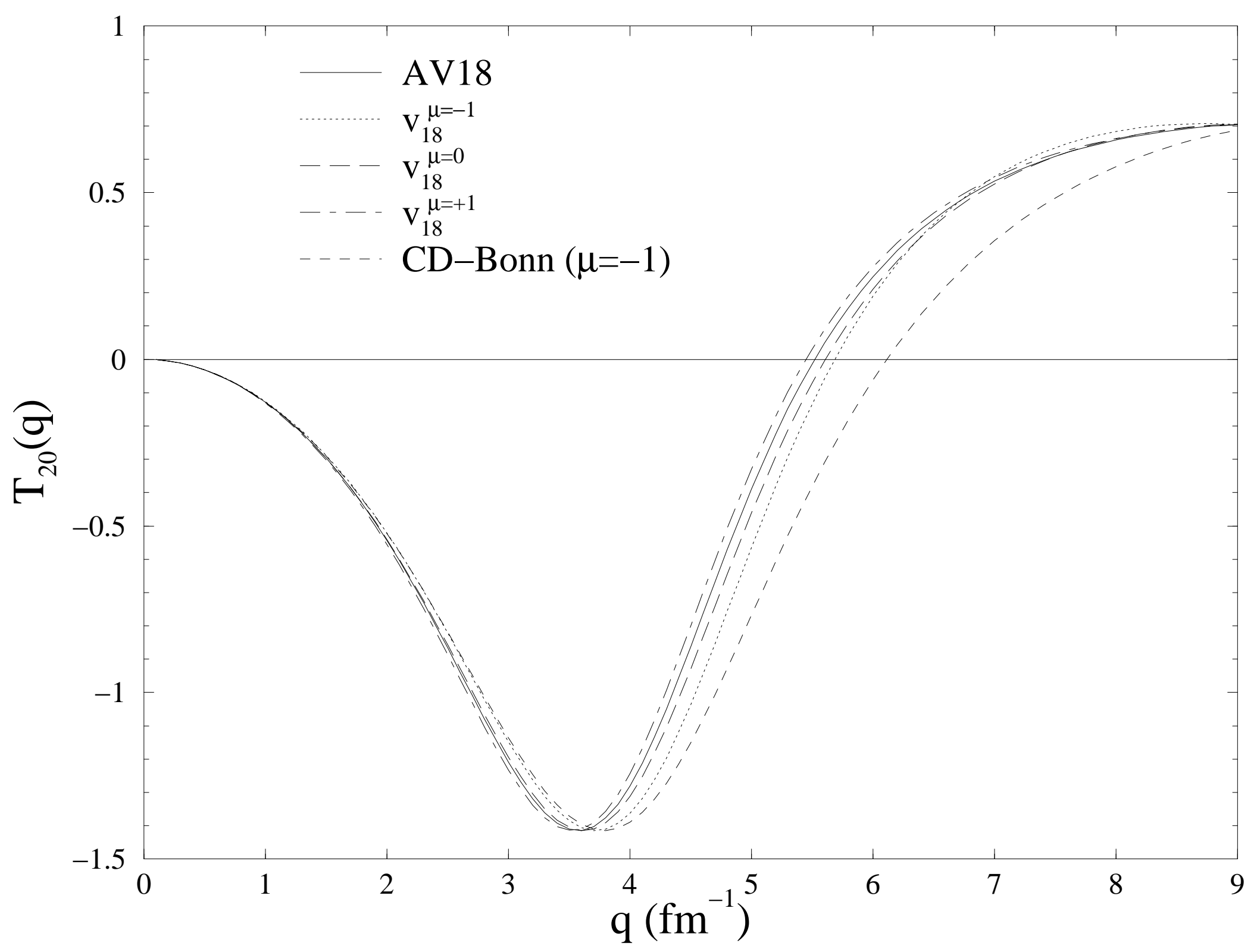




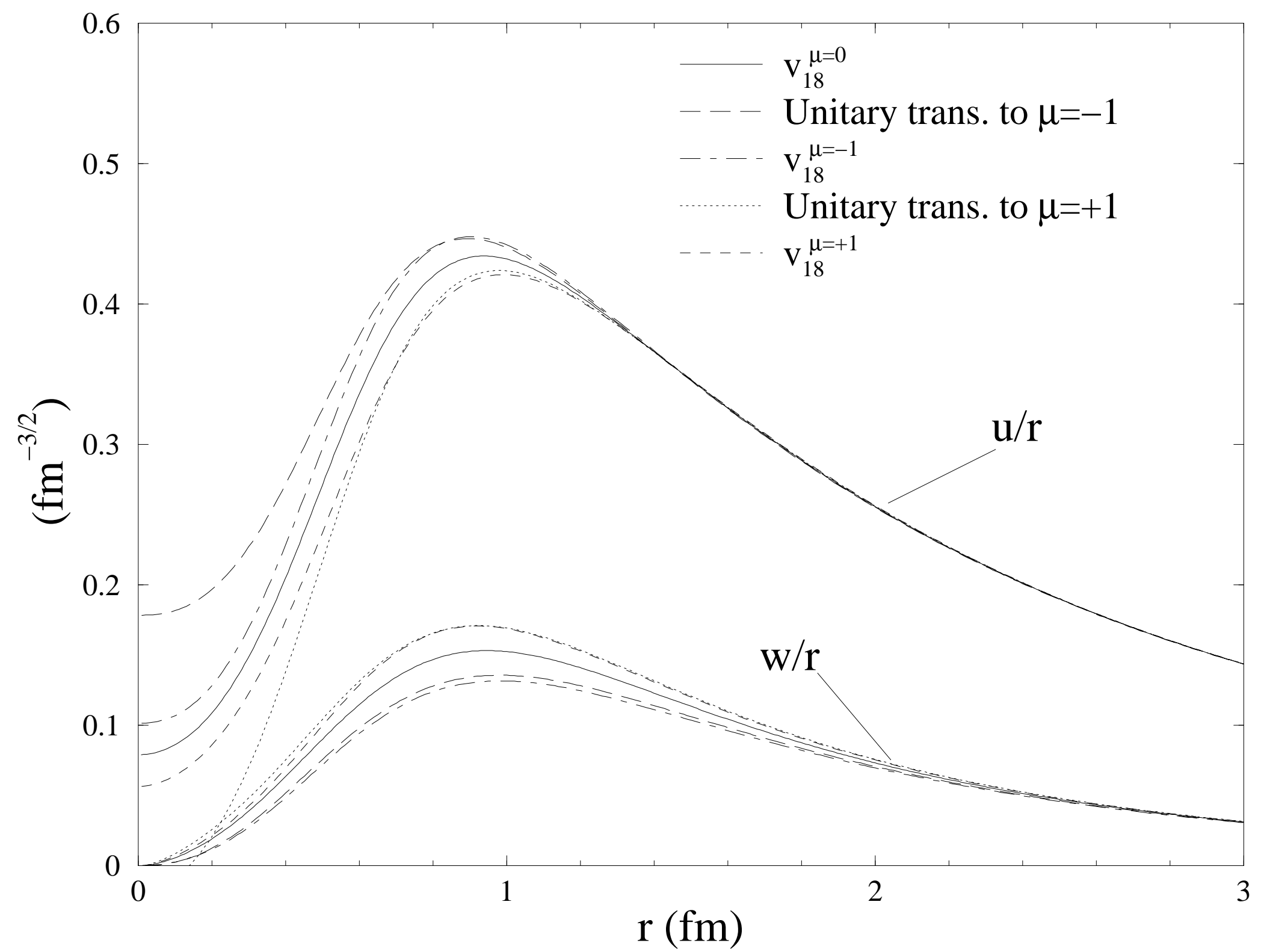




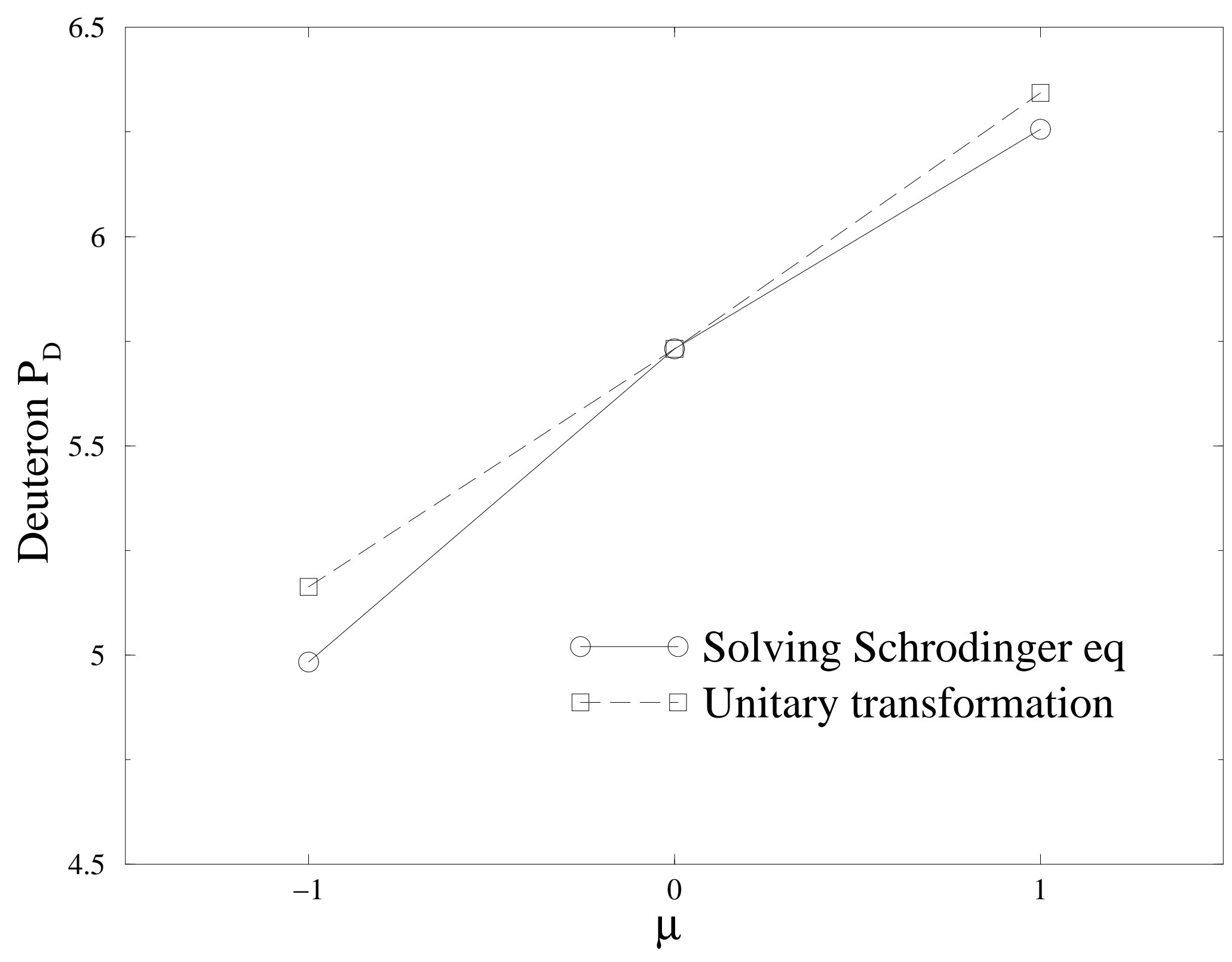




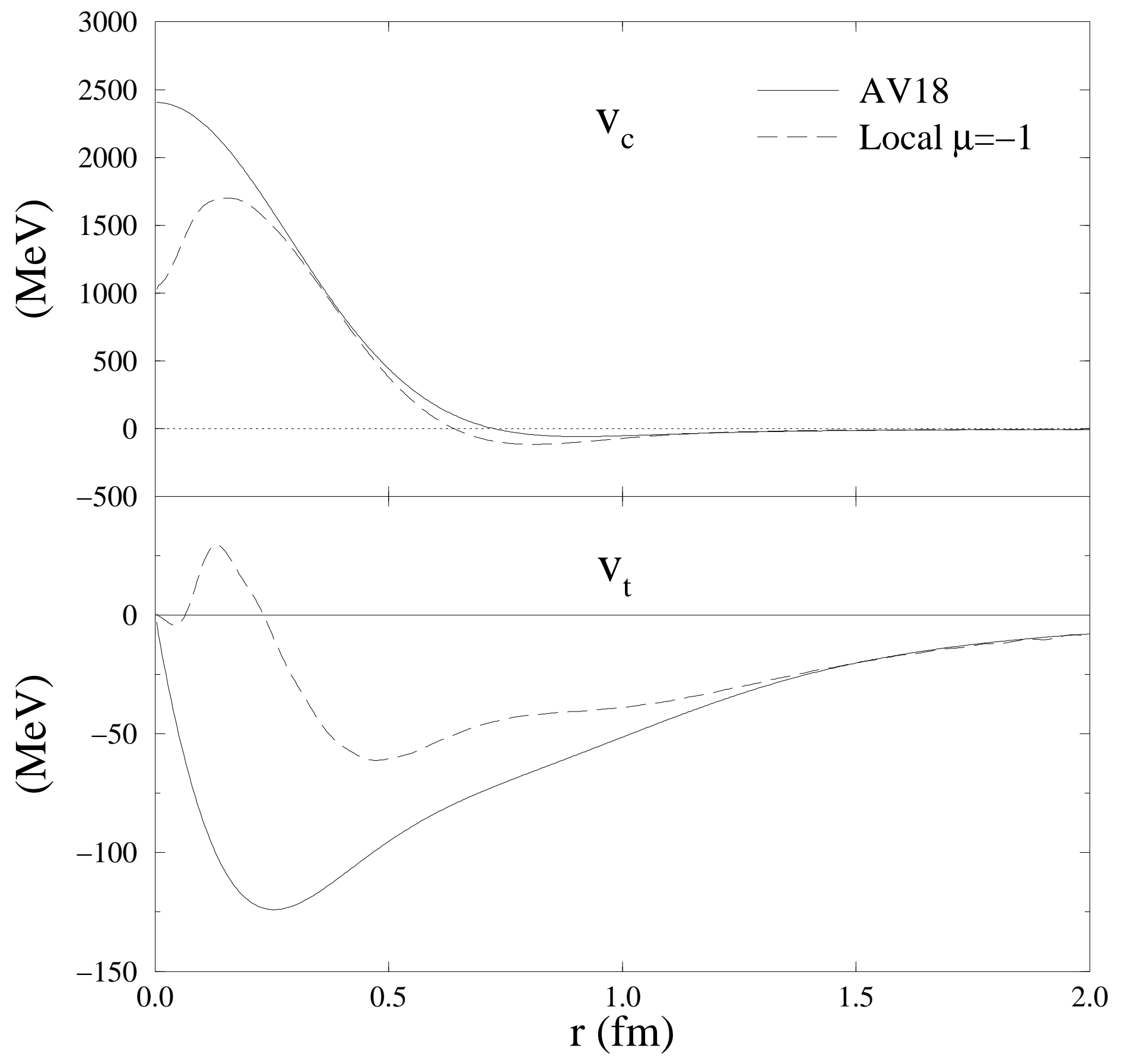

